\title{
Degree constrained orientations in countable graphs
}

\author{
Attila Bernáth* \\ MTA-ELTE Egerváry Research Group (EGRES) \\ Department of Operations Research \\ Eötvös University \\ Pázmány P. s. 1/C \\ H-1117 Budapest, Hungary \\ <bernath@cs.elte.hu>
}

\author{
Henning Bruhn* \\ Mathematisches Seminar \\ Universität Hamburg \\ Bundesstraße 55 \\ 20146 Hamburg, Germany \\ $<$ hbruhn@gmx.net>
}

Submitted: Feb 15, 2007; Accepted: Sep 17, 2008; Published: Sep 29, 2008

Mathematics Subject Classification: 05C20

\begin{abstract}
Degree constrained orientations are orientations of an (undirected) graph where the in-degree function satisfies given lower and upper bounds. For finite graphs Frank and Gyárfás (1976) gave a necessary and sufficient condition for the existence of such an orientation. We extend their result to countable graphs.
\end{abstract}

\section{Introduction}

Orientations of finite graphs are well-studied. An early result is the theorem of Robbins [10] on the existence of a strongly connected orientation. This result has been widely generalised by Nash-Williams [8] in 1960, who described orientations satisfying global or (symmetric) local edge-connectivity requirements. Ford and Fulkerson [4] investigated when a partial orientation can be completed to a di-eulerian one. As a last example, let us cite Frank [5] who characterised the graphs that can be oriented in such a way that there are $k$ directed paths between a specified vertex and every other vertex.

In contrast, not much is known about orientations of infinite graphs. An exception is an old result of Egyed [3] that extends Robbins' theorem on strongly connected orientations. We mention also Thomassen [11] who raised some related conjectures.

In this paper, we will focus on degree constrained orientations in infinite (but countable) graphs. These are orientations where the in-degree function, i.e. the function counting the number of ingoing edges at each vertex, satisfies given lower and upper bounds. Degree constrained orientations have a close relationship to Hall's marriage theorem, and

\footnotetext{
* Supported by the Algorithmic Discrete Optimization Network (ADONET). The first author was also supported by the Hungarian National Foundation for Scientific Research, OTKA grant K 060802.
} 
are also used by Berg and Jordán [1] in the context of graph rigidity. For finite graphs, Frank and Gyárfás [7] gave a necessary and sufficient condition for the existence of a degree constrained orientation. In infinite graphs, however, their condition is no longer sufficient. By strengthening the Frank-Gyárfás condition we will recover sufficiency while maintaining necessity.

Let us briefly recall some standard notation. For subsets $U, W$ of the vertex set of a graph $G=(V, E)$ denote by $i_{G}(U)$ the number of edges in $G$ having both endvertices in $U$ and by $d_{G}(U, W)$ the number of edges in $G$ with one endvertex in $U \backslash W$ and the other in $W \backslash U$. For a directed graph $\vec{G}$ and $X \subseteq V(\vec{G})$ let $\rho_{\vec{G}}(X)$ (resp. $\delta_{\vec{G}}(X)$ ) denote the number of edges entering (resp. leaving) the set $X$. If $x$ is a vertex, we write $\rho_{\vec{G}}(x)$ instead of $\rho_{\vec{G}}(\{x\})$, and if no confusion can arise we will omit the subscripts $G$ and $\vec{G}$. For a function $m: V \rightarrow \mathbb{R}$ and $X \subseteq V$ we will use the notation $m(X)$ to mean $\sum_{x \in X} m(x)$. Unfortunately, this notation is slightly inconsistent, in so far as $\rho_{\vec{G}}(X)$ is, in general, not the same as $\sum_{x \in X} \rho_{\vec{G}}(x)$.

Theorem 1 (Frank and Gyárfás [7]). Let $G=(V, E)$ be a finite graph, and let $l, u: V(G) \rightarrow \mathbb{Z}$ be such that $l(v) \leq u(v)$ for all $v \in V$. Then

(i) there exists an orientation $\vec{G}$ of $G$ such that $l(v) \leq \rho_{\vec{G}}(v) \leq u(v)$ for each vertex $v$ if and only if

(ii) $l(X) \leq i(X)+d(X, V \backslash X)$ and $u(X) \geq i(X)$ for all $X \subseteq V(G)$.

For a proof see also Frank [6].

The result carries over to locally finite graphs (graphs that while possibly infinite have finite degree in each vertex) by an easy compactness argument. For non-locally finite graphs, however, the condition (ii) is too weak for the lower bound, as can be seen by considering an infinite star and setting $l \equiv 1$. There is no orientation satisfying the lower bounds while (ii) clearly holds.

Before we look at this example in more depth, let us rephrase Theorem 1. If we define the surplus to be $s(X)=i(X)+d(X, V \backslash X)-l(X)$ for a graph $G=(V, E)$ and a set $X \subseteq V$, then the theorem states that there is an orientation satisfying the lower bounds if and only if there is no set of negative surplus. Our aim is to find a condition in this vein.

Compare the infinite star with a finite star with the same lower bound of 1 everywhere. The whole finite star has negative surplus of -1 , showing that there is no orientation satisfying the lower bound. Instead of computing this surplus directly let us do it in two steps. First, we observe that the set $L$ of all leaves has surplus $s(L)=0$. Now, if we add the centre $c$ to $L$ we do not gain any new edges since every edge is already incident with a leaf but since $l(c)>0$ the demand for ingoing edges increases. Hence, $L \cup\{c\}$ has negative surplus.

Let us try to do the same for the infinite star. We immediately encounter the problem that the set $L$ of all leaves is incident with infinitely many edges but has infinite demand for ingoing edges, i.e. $l(L)=\infty$. This results in $s(L)=\infty-\infty$, for which it is not clear 
which value this should be. So, let us compute the surplus of $L$ in a similar stepwise fashion as above. Indeed, enumerate the leaves of the infinite star and denote by $L_{n}$ the set of the first $n$ leaves, which then has surplus 0 . As $L$ is the limit of the sets $L_{n}$ it seems justified to define the surplus of $L$ as the limit of $s\left(L_{n}\right)$, which therefore yields 0 . Now, adding the centre $c$ to $L$ we see as for the finite star that the set $L \cup\{c\}$ has negative surplus. Consequently, the set $L \cup\{c\}$ is a witness for the non-existence of an orientation respecting the lower bounds.

We will now turn the ad hoc reasoning in the preceding paragraph into a formal condition. Fix a graph $G=(V, E)$, and for an ordinal number $\theta$ call a family $\mathcal{U}_{\theta}:=\left(U_{\mu}\right)_{\mu \leq \theta}$ of subsets of $V$ a queue in $G$ if

- $U_{0}=\emptyset$;

- $U_{\mu} \subseteq U_{\lambda}$ for all $\mu \leq \lambda \leq \theta$;

- $U_{\lambda}=\bigcup_{\mu<\lambda} U_{\mu}$ for each limit ordinal $\lambda \leq \theta$.

We write $\mathcal{U}_{\lambda}$ for the initial segment up to $\lambda$ of $\mathcal{U}_{\theta}$, i.e. $\mathcal{U}_{\lambda}=\left(U_{\mu}\right)_{\mu \leq \lambda}$.

Let $l: V \rightarrow \mathbb{Z}$ be a non-negative function, and let $\mathcal{U}_{\theta}=\left(U_{\lambda}\right)_{\lambda \leq \theta}$ be a queue in $G$. Putting $\eta\left(\mathcal{U}_{0}, l\right)=0$, we define by transfinite induction a function $\eta$ such that

$$
\eta\left(\mathcal{U}_{\lambda+1}, l\right)=\eta\left(\mathcal{U}_{\lambda}, l\right)+i\left(U_{\lambda+1} \backslash U_{\lambda}\right)+d\left(U_{\lambda+1} \backslash U_{\lambda}, V \backslash U_{\lambda+1}\right)-l\left(U_{\lambda+1} \backslash U_{\lambda}\right)
$$

and such that $\eta\left(\mathcal{U}_{\lambda}, l\right)=\liminf _{\mu<\lambda} \eta\left(\mathcal{U}_{\mu}, l\right)$ for limit ordinals $\lambda$. In the computation of $\eta$ we might need to calculate with $\infty$; we use the convention that $\infty-\infty=\infty$. Sometimes, if confusion can arise, we will write $\eta_{G}$ to specify the underlying graph. We remark that for a finite vertex set the $\eta$-function provides merely an overly complicated way of computing its surplus. For infinite sets, however, $\eta$ can be seen as a refinement of the surplus.

A set $U \subseteq V$ will be called $l$-deficient (or simply deficient if $l$ is clear from the context) if there exists a queue $\mathcal{U}_{\theta}=\left(U_{\lambda}\right)_{\lambda \leq \theta}$ with $U=U_{\theta}$ and $\eta\left(\mathcal{U}_{\theta}, l\right)<0$. Deficient sets will play the same role as sets of negative surplus in the finite case.

We can now state our main result, which we will prove in the next section:

Theorem 2. Let $G=(V, E)$ be a countable graph, and let $l, u: V \rightarrow \mathbb{Z} \cup\{\infty\}$ be non-negative functions with $l \leq u$. Then the following statements are equivalent:

(i) there exists an orientation $\vec{G}$ of $G$ such that $l(v) \leq \rho_{\vec{G}}(v) \leq u(v)$ for each vertex $v$; and

(ii) there are no l-deficient sets and $u(X) \geq i(X)$ for all finite $X \subseteq V(G)$.

We mention that the theorem is very much in spirit of [9], in which Nash-Williams extends Hall's marriage theorem to countable graphs. This is perhaps not at all surprising since for finite graphs Theorem 1 can be reduced to the marriage theorem and vice versa. For infinite graphs, there are several versions of Hall's theorem. From the one in [9] one can indeed obtain our main result. However, as our proof is not a simple translation of 
Nash-Williams' arguments and as the reduction of Theorem 2 to Nash-Williams' theorem is not at all immediate (it takes about two pages), we see merit in providing a direct proof.

Nash-Williams' idea to refine a finite condition by using transfinite sequences is also used in Wojciechowski [12], who investigates when an infinite family of matroids on the same ground set has a system of disjoint bases.

\section{Proof of main result}

The graphs we consider are allowed to have parallel edges and loops and may be infinite. For general graph theoretic notation and terms we refer the reader to Diestel [2]. In this section $G$ will always denote a graph with vertex set $V$ and edge set $E$, and $l, u: V \rightarrow$ $\mathbb{Z} \cup\{\infty\}$ will always be non-negative functions such that $l \leq u$.

We shall prove the main result in the course of this section. Let us start with the observation that the function $\eta$ satisfies a submodularity-type inequality (a set function $b: 2^{V} \rightarrow \mathbb{R}$ is called submodular if $b(X)+b(Y) \geq b(X \cap Y)+b(X \cup Y)$ for any $\left.X, Y \subseteq V\right)$. More precisely, it is easy to see that for the surplus function it holds that

$$
s(U)+s(W)=s(U \cup W)+s(U \cap W)+d(U \backslash W, W \backslash U),
$$

where $U, W$ are vertex sets. (Clearly, this implies that $s$ is submodular.) The lemma below states that a similar relation is true for $\eta$.

For a set $X \subseteq V$, we will denote its complement $V \backslash X$ by $\bar{X}$ if the base set $V$ is clear from the context. To ease notation further, we will say that $\mathcal{U}=\left(U_{\lambda}\right)_{\lambda \leq \theta}$ is a queue for a set $U$ if $U=U_{\theta}$. For a successor ordinal $\lambda$, we write (slightly abusing notation) $\lambda-1$ for the ordinal $\mu$ for which $\lambda=\mu+1$. We also introduce the notation $U_{\lambda}^{\prime}:=U_{\lambda} \backslash U_{\lambda-1}$.

Lemma 3. Let $\mathcal{U}=\left(U_{\lambda}\right)_{\lambda \leq \theta}$ be a queue for $U$ and $\mathcal{W}=\left(W_{\lambda}\right)_{\lambda \leq \kappa}$ be a queue for $W$. Define queues $\mathcal{X}=\left(X_{\lambda}\right)_{\lambda \leq \kappa}$ with $X_{\lambda}=U \cap W_{\lambda}$ for every $\lambda \leq \kappa$ and $\mathcal{Y}=\left(Y_{\lambda}\right)_{\lambda \leq \theta+\kappa}$ with $Y_{\lambda}=U_{\lambda}$ for $\lambda \leq \theta$ and $Y_{\theta+\lambda}=U \cup W_{\lambda}$ for $\lambda \leq \kappa$. Then

$$
\eta(\mathcal{U}, l)+\eta(\mathcal{W}, l) \geq \eta(\mathcal{X}, l)+\eta(\mathcal{Y}, l)+d(W \backslash U, U \backslash W)
$$

Proof. We shall show that

$$
\eta(\mathcal{U}, l)+\eta\left(\mathcal{W}_{\lambda}, l\right) \geq \eta\left(\mathcal{X}_{\lambda}, l\right)+\eta\left(\mathcal{Y}_{\theta+\lambda}, l\right)+d\left(W_{\lambda} \backslash U, U \backslash W_{\lambda}\right)
$$

for all $\lambda \leq \kappa$, which will give the statement with $\lambda=\kappa$.

We have $\eta\left(\mathcal{W}_{0}, l\right)=\eta\left(\mathcal{X}_{0}, l\right)=0, \eta\left(\mathcal{Y}_{\theta}, l\right)=\eta(\mathcal{U}, l)$ and $d\left(W_{0} \backslash U, U \backslash W_{0}\right)=0$ since $W_{0}=\emptyset$. Therefore, (1) holds with equality for $\lambda=0$. We proceed by transfinite induction. Let $\lambda$ be the smallest ordinal for which (1) is not yet shown.

First, assume $\lambda$ to be a successor ordinal. Observe that

$$
d_{x}:=d\left(X_{\lambda}^{\prime}, \overline{X_{\lambda}}\right)=d\left(X_{\lambda}^{\prime}, W_{\lambda} \backslash U\right)+d\left(X_{\lambda}^{\prime}, \overline{W_{\lambda}}\right)
$$




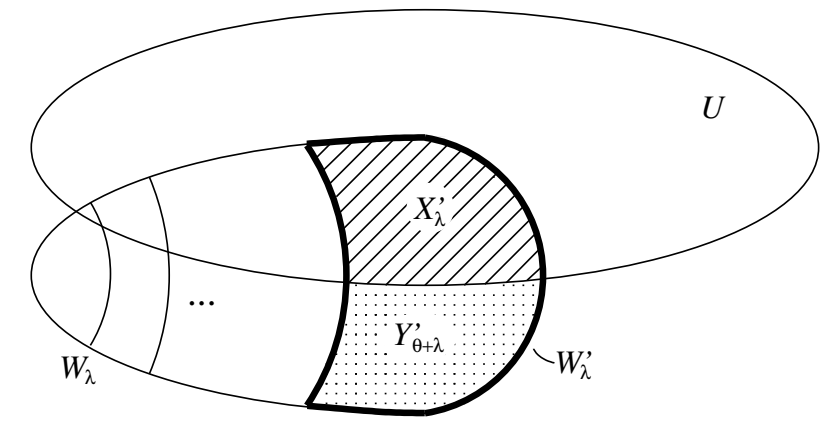

Figure 1: Relevant sets in Lemma 3

and

$$
d_{y}:=d\left(Y_{\theta+\lambda}^{\prime}, \overline{Y_{\theta+\lambda}}\right)=d\left(W_{\lambda}^{\prime} \backslash U, \overline{U \cup W_{\lambda}}\right) .
$$

We use these two relations in what follows:

$$
\begin{aligned}
d\left(X_{\lambda}^{\prime}, W_{\lambda} \backslash U\right)+d\left(W_{\lambda}^{\prime}, \overline{W_{\lambda}}\right) \\
=\quad d\left(X_{\lambda}^{\prime}, W_{\lambda} \backslash U\right)+d\left(Y_{\theta+\lambda}^{\prime}, \overline{Y_{\theta+\lambda}}\right) \\
\quad+d\left(Y_{\theta+\lambda}^{\prime}, U \backslash W_{\lambda}\right)+d\left(X_{\lambda}^{\prime}, \overline{W_{\lambda}}\right) \\
=\quad d_{x}+d_{y}+d\left(Y_{\theta+\lambda}^{\prime}, U \backslash W_{\lambda}\right)
\end{aligned}
$$

Noting that

$$
i\left(W_{\lambda}^{\prime}\right)=i\left(X_{\lambda}^{\prime}\right)+i\left(Y_{\theta+\lambda}^{\prime}\right)+d\left(X_{\lambda}^{\prime}, Y_{\theta+\lambda}^{\prime}\right)
$$

and that

$$
d\left(X_{\lambda}^{\prime}, W_{\lambda} \backslash U\right)=d\left(X_{\lambda}^{\prime}, Y_{\theta+\lambda}^{\prime}\right)+d\left(X_{\lambda}^{\prime}, W_{\lambda-1} \backslash U\right)
$$

we obtain

$$
\begin{aligned}
& d\left(X_{\lambda}^{\prime}, W_{\lambda-1} \backslash U\right)+d\left(W_{\lambda}^{\prime}, \overline{W_{\lambda}}\right)+i\left(W_{\lambda}^{\prime}\right) \\
& \quad=d_{x}+i\left(X_{\lambda}^{\prime}\right)+d_{y}+i\left(Y_{\theta+\lambda}^{\prime}\right)+d\left(Y_{\theta+\lambda}^{\prime}, U \backslash W_{\lambda}\right)
\end{aligned}
$$

Using this and the induction hypothesis for $\lambda-1$ we get

$$
\begin{aligned}
\eta(\mathcal{U}, l)+\eta\left(\mathcal{W}_{\lambda}, l\right)= & \eta(\mathcal{U}, l)+\eta\left(\mathcal{W}_{\lambda-1}, l\right)+d\left(W_{\lambda}^{\prime}, \overline{W_{\lambda}}\right)+i\left(W_{\lambda}^{\prime}\right)-l\left(W_{\lambda}^{\prime}\right) \\
\stackrel{(1)}{\geq} & \eta\left(\mathcal{X}_{\lambda-1}, l\right)+\eta\left(\mathcal{Y}_{\theta+(\lambda-1)}, l\right)+d\left(W_{\lambda-1} \backslash U, U \backslash W_{\lambda-1}\right) \\
& +d\left(W_{\lambda}^{\prime}, \overline{W_{\lambda}}\right)+i\left(W_{\lambda}^{\prime}\right)-l\left(W_{\lambda}^{\prime}\right) \\
= & \eta\left(\mathcal{X}_{\lambda-1}, l\right)+\eta\left(\mathcal{Y}_{\theta+(\lambda-1)}, l\right)+d\left(W_{\lambda-1} \backslash U, U \backslash W_{\lambda}\right) \\
& +d\left(W_{\lambda-1} \backslash U, X_{\lambda}^{\prime}\right)+d\left(W_{\lambda}^{\prime}, \overline{W_{\lambda}}\right)+i\left(W_{\lambda}^{\prime}\right)-l\left(W_{\lambda}^{\prime}\right) \\
\stackrel{(2)}{=} & \eta\left(\mathcal{X}_{\lambda-1}, l\right)+d_{x}+i\left(X_{\lambda}^{\prime}\right)-l\left(X_{\lambda}^{\prime}\right) \\
& +\eta\left(\mathcal{Y}_{\theta+(\lambda-1)}, l\right)+d_{y}+i\left(Y_{\theta+\lambda}^{\prime}\right)-l\left(Y_{\theta+\lambda}^{\prime}\right) \\
& +d\left(W_{\lambda-1} \backslash U, U \backslash W_{\lambda}\right)+d\left(Y_{\theta+\lambda}^{\prime}, U \backslash W_{\lambda}\right) \\
= & \eta\left(\mathcal{X}_{\lambda}, l\right)+\eta\left(\mathcal{Y}_{\theta+\lambda}, l\right)+d\left(W_{\lambda} \backslash U, U \backslash W_{\lambda}\right) .
\end{aligned}
$$


This proves the induction step when $\lambda$ is a successor ordinal.

Second, assume that $\lambda$ is a limit ordinal. Then

$$
\begin{aligned}
\eta(\mathcal{U}, l)+\eta\left(\mathcal{W}_{\lambda}, l\right) & =\eta(\mathcal{U}, l)+\lim \inf _{\mu<\lambda} \eta\left(\mathcal{W}_{\mu}, l\right) \\
& \geq \lim \inf _{\mu<\lambda}\left(\eta\left(\mathcal{X}_{\mu}, l\right)+\eta\left(\mathcal{Y}_{\theta+\mu}, l\right)+d\left(W_{\mu} \backslash U, U \backslash W_{\mu}\right)\right) \\
& \geq \eta\left(\mathcal{X}_{\lambda}, l\right)+\eta\left(\mathcal{Y}_{\theta+\lambda}, l\right)+\liminf _{\mu<\lambda} d\left(W_{\mu} \backslash U, U \backslash W_{\mu}\right) .
\end{aligned}
$$

Furthermore, for any $\mu<\lambda$ we get

$$
\begin{aligned}
d\left(W_{\mu} \backslash U, U \backslash W_{\mu}\right) & =d\left(W_{\mu} \backslash U, U \backslash W_{\lambda}\right)+d\left(W_{\mu} \backslash U, U \cap\left(W_{\lambda} \backslash W_{\mu}\right)\right) \\
& \geq d\left(W_{\mu} \backslash U, U \backslash W_{\lambda}\right) .
\end{aligned}
$$

It is easy to see that $\liminf _{\mu<\lambda} d\left(W_{\mu} \backslash U, U \backslash W_{\lambda}\right)=d\left(W_{\lambda} \backslash U, U \backslash W_{\lambda}\right)$ since $W_{\lambda}=\bigcup_{\mu<\lambda} W_{\mu}$. Putting all this together we obtain (1).

We call a vertex set $U$ l-tight if (it is not $l$-deficient and) there exists a queue $\left(U_{\lambda}\right)_{\lambda \leq \theta}$ for $U$ with $\eta\left(\mathcal{U}_{\theta}, l\right)=0$. If it is clear in regard to which function $l$ a set is tight, we will suppress the $l$. Tight sets are the most critical sets, and it can be seen that in an orientation respecting the lower bound $l$ there can be no edge leaving a tight set.

Lemma 3 immediately implies that the intersection and the union of two tight sets is tight, too. We will need a little bit more, namely that this also holds for the union of countably many tight sets:

Lemma 4. Assume that there are no deficient sets in $G$, and let $U_{1}, U_{2}, \ldots$ be countably many tight sets. Then also their union is tight.

Proof. Let $\mathcal{U}^{i}=\left(U_{\lambda}^{i}\right)_{\lambda \leq \theta_{i}}$ be queues witnessing the tightness of $U_{i}$ for each $i$, i.e. $\eta\left(\mathcal{U}^{i}, l\right)=$ 0 and $U_{\theta_{i}}^{i}=U_{i}$. For any $n \in \mathbb{N}$, set $\kappa_{n}=\sum_{i=1}^{n} \theta_{i}$ and $\kappa=\sum_{i=1}^{\infty} \theta_{i}$. Then we can define the queue $\mathcal{Y}=\left(Y_{\lambda}\right)_{\lambda \leq \kappa}$ with $Y_{\kappa_{n-1}+\lambda}=Y_{\kappa_{n-1}} \cup U_{\lambda}^{n}$ if $\lambda \leq \theta_{n}$ (where $\kappa_{0}=0$ and $Y_{0}=\emptyset$ ) and $Y_{\kappa}=\bigcup_{n=1}^{\infty} Y_{\kappa_{n}}$. By Lemma 3 and induction we get $\eta\left(\mathcal{Y}_{\kappa_{n}}, l\right)=0$ for all $n \geq 0$ :

$$
\eta\left(\mathcal{Y}_{\kappa_{n}}, l\right) \leq \eta\left(\mathcal{Y}_{\kappa_{n-1}}, l\right)+\eta\left(\mathcal{U}^{n}, l\right)=0+0
$$

(Note, that there are no deficient sets.) From this it follows that

$$
\eta(\mathcal{Y}, l)=\lim \inf _{\lambda<\kappa} \eta\left(\mathcal{Y}_{\lambda}, l\right) \leq 0
$$

Again, as there are no deficient sets, this implies that $Y_{\kappa}=\bigcup_{i=1}^{\infty} U_{i}$ is tight.

As for the lower bound we will define deficiency and tightness of sets with respect to the upper bound, too. We call a finite vertex set $X u$-faulty, if $u(X)-i(X)<0$, and we call it $u$-taut if $u(X)-i(X)=0$. Again, if $u$ is clear from the context, we will omit it.

In the last lemma we saw that the union of tight sets is tight. In contrast, for taut sets we will need that their intersection is taut: 
Lemma 5. If there are no faulty sets in $G$ then the following is true:

(i) if $X$ and $Y$ are two taut sets then $X \cap Y$ is taut and there is no edge between $X \backslash Y$ and $Y \backslash X$; and

(ii) the intersection of arbitrarily many taut sets is taut.

Proof. (i) On the one hand, we get

$$
i(X)+i(Y)=u(X)+u(Y)=u(X \cup Y)+u(X \cap Y) \geq i(X \cup Y)+i(X \cap Y)
$$

and on the other hand, $i$ is supermodular, i.e. it holds that:

$$
i(X)+i(Y) \leq i(X \cup Y)+i(X \cap Y) .
$$

Thus, we have equality everywhere. In particular, if there was an edge between $X \backslash Y$ and $Y \backslash X$ then $i(X)+i(Y)<i(X \cup Y)+i(X \cap Y)$, which is not the case.

(ii) Let $X_{i}, i \in I$ be taut sets. Since by definition each of the $X_{i}$ is finite, their intersection is also finite. Hence, there are already finitely many $X_{j}, j \in J \subseteq I$ with $\bigcap_{i \in I} X_{i}=\bigcap_{j \in J} X_{j}$. Therefore, we only need to check that the intersection of two taut sets is taut, which is true by (i).

In Theorem 2 (ii) the conditions regarding the lower and the upper bound are independent of each other. The following lemma provides a link between tight and taut sets.

Lemma 6. Let there be neither deficient sets nor faulty sets in $G$, and let $U$ be a taut set and $L$ be a tight set. Then $U \backslash L$ is taut and $L \backslash U$ is tight.

Proof. Since the proof is technical but not too hard, we only give an indication of how the lemma is proved.

Let $\mathcal{L}=\left(L_{\lambda}\right)_{\lambda \leq \theta}$ be a queue with $\eta(\mathcal{L}, l)=0$ and $L_{\theta}=L$, and define $\mathcal{M}=\left(L_{\lambda} \backslash U\right)_{\lambda \leq \theta}$. Using transfinite induction and arguments similar to those in the proof of Lemma 3 , one can show that for any ordinal $\lambda \leq \theta$ we have

$$
\eta\left(\mathcal{L}_{\lambda}, l\right) \geq \eta\left(\mathcal{M}_{\lambda}, l\right)+i\left(L_{\lambda} \cap U\right)-l\left(L_{\lambda} \cap U\right)+d\left(L_{\lambda} \cap U, \overline{L_{\lambda}}\right) .
$$

Now, for $\lambda=\theta$ this yields

$$
\begin{aligned}
0 & =\eta(\mathcal{L}, l)+u(U)-i(U) \\
& \geq \eta(\mathcal{M}, l)+i(L \cap U)-l(L \cap U)+u(U)-i(U)+d(L \cap U, \bar{L}) \\
& \geq \eta(\mathcal{M}, l)+u(U \backslash L)-i(U \backslash L)+(u-l)(L \cap U)
\end{aligned}
$$

Since $\eta(\mathcal{M}, l) \geq 0, u \geq l$ and since $u(U \backslash L) \geq i(U \backslash L)$ it follows that $U \backslash L$ is taut. This then also implies that $\eta(\mathcal{M}, l)=0$, and hence $L \backslash U$ is tight. 
Lemma 7. Let there be no u-faulty sets. Assume that for an edge e with endvertices $v, w$ there is no u-taut set $U$ with $v \in U$ but $w \notin U$. Then, setting $u^{\prime}(x)=u(x)$ for all vertices $x \neq v$ and $u^{\prime}(v)=u(v)-1$, there are no $u^{\prime}$-faulty sets in $G-e$.

Proof. If $u(v)=\infty$ then for every set $X \subseteq V$ we get $u(X)=\infty$, and thus there cannot be any $u^{\prime}$-faulty set in $G-e$. So, let $u(v)<\infty$, and suppose $U$ is $u^{\prime}$-faulty in $G-e$. Clearly, $v \in U$ but $w \notin U$ since there are no $u$-faulty sets in $G$. But then $U$ is $u$-taut in $G$, a contradiction.

We can finally prove our main result:

Proof of Theorem 2. (ii) $\Rightarrow$ (i) Let $v_{1}, v_{2}, \ldots$ be a sequence of the vertices of $G$ such that every vertex $v$ appears exactly $l(v)$ times in it. Putting $l_{0}=l$ and $u_{0}=u$ we recursively

(a) set $l_{n}(v)=l_{n-1}(v)$ if $v \neq v_{n}$ and $l_{n}\left(v_{n}\right)=l_{n-1}\left(v_{n}\right)-1$;

(b) set $u_{n}(v)=u_{n-1}(v)$ if $v \neq v_{n}$ and $u_{n}\left(v_{n}\right)=u_{n-1}\left(v_{n}\right)-1$; and

(c) find distinct edges $e_{1}, e_{2}, \ldots$ such that $G_{n}:=G-\left\{e_{1}, \ldots, e_{n}\right\}$ has no $l_{n}$-deficient and no $u_{n}$-faulty sets and such that $e_{n}$ is incident with $v_{n}$.

Assume that this has been achieved for $i<n$. It is not difficult to check directly that picking any loop at $v_{n}$ for $e_{n}$ we satisfy (a)-(c). However, if we agree that $v_{n}$ is a neighbour of itself if there is a loop at $v_{n}$ then what follows covers also loops.

For each neighbour $w$ of $v_{n}$ in $G_{n-1}$ for which this is possible pick an $l_{n-1}$-tight set $X$ with $w \in X$ but $v_{n} \notin X$, and consider the union $L$ of these sets. By Lemma $4, L$ is still $l_{n-1}$-tight. In a similar way, consider a minimal $u_{n-1}$-taut set $U$ that contains $v_{n}$ (where we set $U=\emptyset$ if there is no such set). From Lemma 5 (ii) it follows that for a neighbour $w$ of $v_{n}$ in $G_{n-1}$ for which there is an $u_{n-1}$-taut set $Y$ with $v_{n} \in Y$ but $w \notin Y$ it holds that $w \notin U$. By Lemma $6, U \backslash L$ is $u_{n-1}$-taut, too. As $U$ is minimal this implies $U=U \backslash L$ and therefore that $U$ and $L$ are disjoint.

Next, if $U \neq \emptyset$ then there is a neighbour $w_{n}$ of $v_{n}$ in $G_{n-1}$ with $w_{n} \in U$. For if that was not the case, then, recalling that $u\left(v_{n}\right)>0$ by definition of $v_{n}$, we would have

$$
i\left(U \backslash\left\{v_{n}\right\}\right)=i(U)=u(U)>u\left(U \backslash\left\{v_{n}\right\}\right),
$$

which is a contradiction, as there are no $u_{n-1}$-faulty sets. Note that $w_{n} \notin L$ since $U$ and $L$ are disjoint. If, on the other hand, $U=\emptyset$ then there is a neighbour $w_{n} \notin L$ of $v_{n}$ in $G_{n-1}$. Indeed, suppose not. Let $\mathcal{L}:=\left(L_{\lambda}\right)_{\lambda \leq \theta}$ be a queue with $\eta\left(\mathcal{L}, l_{n-1}\right)=0$ and $L_{\theta}=L$ (recall, that $L$ is $l_{n-1}$-tight). Put $L_{\theta+1}=L \cup\left\{v_{n}\right\}$, and observe that $i\left(L_{\theta+1} \backslash L_{\theta}\right)=0$ (since there is no loop at $\left.v_{n}\right)$ and $d_{G_{n-1}}\left(L_{\theta+1} \backslash L_{\theta}, V\left(G_{n-1}\right) \backslash L_{\theta+1}\right)=0$. Thus, $\eta\left(\mathcal{L}_{\theta+1}, l_{n-1}\right)=-l_{n-1}\left(v_{n}\right)<0$, (from the definition of our sequence $v_{1}, v_{2}, \ldots$ it follows that $\left.l_{n-1}\left(v_{n}\right)>0\right)$. Consequently, $L_{\theta+1}$ is $l_{n-1}$-deficient, contrary to our induction hypothesis. In any case, let $e_{n}$ be any edge between $v_{n}$ and $w_{n}$ and observe that, by Lemma 7 , there are no $u_{n}$-faulty sets in $G_{n}$. In addition, by construction of $L$ and because of $w_{n} \notin L$ we get

$$
\text { there is no } l_{n-1} \text {-tight set } X \text { in } G_{n-1} \text { with } w_{n} \in X \text { but } v_{n} \notin X
$$


Let us check that there are also no $l_{n}$-deficient sets in $G_{n}$. So, suppose there is a $l_{n}$-deficient set $M$ in $G_{n}$, and let $\mathcal{M}_{\theta}=\left(U_{\lambda}\right)_{\lambda \leq \theta}$ be a queue in $G_{n}$ with $M=M_{\theta}$ and $\eta_{G_{n}}\left(\mathcal{M}_{\theta}, l_{n}\right)<0$. Since $G_{n}$ differs from $G_{n-1}$ only in the edge $e_{n}$ we get, if neither $v_{n} \in M$ nor $w_{n} \in M$, that $\eta_{G_{n-1}}\left(\mathcal{M}_{\theta}, l_{n-1}\right)=\eta_{G_{n}}\left(\mathcal{M}_{\theta}, l_{n}\right)$, which is impossible since $M$ is not $l_{n-1}$-deficient in $G_{n-1}$. In a similar way, if $l_{n-1}\left(v_{n}\right)<\infty$ we can exclude the case when $v_{n} \in M$ as we lose an edge but also have less demand of ingoing edges. If, on the other hand, $l_{n-1}\left(v_{n}\right)=\infty$ we can get rid of this case, too: Denote by $\lambda$ the smallest ordinal for which $v_{n} \in M_{\lambda}$, which is a successor ordinal, by definition of a queue. Then as $\eta_{G_{n-1}}\left(\mathcal{M}_{\lambda}, l_{n-1}\right) \geq 0$ and as

$$
\eta_{G_{n-1}}\left(\mathcal{M}_{\lambda}, l_{n-1}\right)=\eta_{G_{n-1}}\left(\mathcal{M}_{\lambda-1}, l_{n-1}\right)+i\left(M_{\lambda}^{\prime}\right)+d\left(M_{\lambda}^{\prime}, \overline{M_{\lambda}}\right)-\infty
$$

it follows that $\eta_{G_{n-1}}\left(\mathcal{M}_{\lambda}, l_{n-1}\right)=\infty$, and hence $\eta_{G_{n-1}}\left(\mathcal{M}_{\theta}, l_{n-1}\right)=\infty$. Because $\eta_{G_{n-1}}$ and $\eta_{G_{n}}$ can differ by at most one, we obtain $\eta_{G_{n}}\left(\mathcal{M}_{\theta}, l_{n}\right)=\infty$, a contradiction.

Therefore, we may assume that $w_{n} \in M$ but $v_{n} \notin M$ (independent of the value of $\left.l_{n-1}\left(v_{n}\right)\right)$. Now, let $\lambda$ be the smallest ordinal for which $w_{n} \in M_{\lambda}$, which is a successor ordinal. Then,

$$
d_{G_{n-1}}\left(M_{\lambda} \backslash M_{\lambda-1}, \overline{M_{\lambda}}\right)=d_{G_{n}}\left(M_{\lambda} \backslash M_{\lambda-1}, \overline{M_{\lambda}}\right)+1
$$

and thus $\eta_{G_{n-1}}\left(\mathcal{M}_{\lambda}, l_{n-1}\right)=\eta_{G_{n}}\left(\mathcal{M}_{\lambda}, l_{n}\right)+1$ (since $v_{n} \notin M_{\lambda}$ implies that $l_{n-1}\left(M_{\lambda} \backslash M_{\lambda-1}\right)=$ $\left.l_{n}\left(M_{\lambda} \backslash M_{\lambda-1}\right)\right)$. Hence, $\eta_{G_{n-1}}\left(\mathcal{M}_{\theta}, l_{n-1}\right)=\eta_{G_{n}}\left(\mathcal{M}_{\theta}, l_{n}\right)+1$. Now, since $\eta_{G_{n}}\left(\mathcal{M}_{\theta}, l_{n}\right)<0$ but $\eta_{G_{n-1}}\left(\mathcal{M}_{\theta}, l_{n-1}\right) \geq 0$ we obtain that $\eta_{G_{n-1}}\left(\mathcal{M}_{\theta}, l_{n-1}\right)=0$. Therefore, $M$ is an $l_{n-1^{-}}$ tight set with $v_{n} \notin M$ but $w_{n} \in M$, contradicting (3). Thus, there are no $l_{n}$-deficient sets in $G_{n}$, as required.

Having terminated the transfinite induction, we put $G^{0}=G-\left\{e_{1}, e_{2}, \ldots\right\}$. We think of each edge $e_{n}$ as already directed towards $v_{n}$. In this way, each vertex $v$ has an indegree of exactly $l(v)$ (by definition of the vertex enumeration). So, what remains is to direct the edges in $G^{0}$ in such a way, that the reduced upper bound $u^{0}:=u-l$ is respected.

First, let us show that there are no $u^{0}$-faulty sets in $G^{0}$. Indeed, consider a finite vertex set $U$ in $G^{0}$. Then there is an $N$ such that $u_{N}(U)=u^{0}(U)$ and $i_{G_{N}}(U)=i_{G^{0}}(U)$, and thus $u^{0}(U) \geq i_{G^{0}}(U)$ since $u_{N}(U) \geq i_{G_{N}}(U)$. As a $u^{0}$-faulty set is by definition finite, there is therefore no such set.

Second, let $f_{1}, f_{2}, \ldots$ be an enumeration of the edges of $G^{0}$. Denote the endvertices of $f_{1}$ by $x$ and $y$, and observe that if there is a $u^{0}$-taut set $X$ with $x \in X$ but $y \notin X$ then there is no $u^{0}$-taut set $Y$ with $x \notin Y$ but $y \in Y$, by Lemma 5 (i).

Now, if there is such a set $X$, then direct $f_{1}$ towards $y$, and define $u^{1}(v)=u^{0}(v)$ for $v \neq y$ and $u^{1}(y)=u^{0}(y)-1$. If not, direct $f_{1}$ in the other way, and define $u^{1}$ accordingly. Lemma 7 ensures that $G^{1}=G^{0}-f_{1}$ has no $u^{1}$-faulty sets. Continuing in this way, we obtain the desired orientation. Indeed, suppose a vertex $v$ receives more ingoing edges than $u(v)$. Then there is an $N$ such that $u^{N}(v)<0$, which implies that $\{v\}$ is $u^{N}$-faulty, a contradiction.

(i) $\Rightarrow$ (ii) Let $\vec{G}$ be an orientation as in (i). Then trivially $u(X) \geq \sum_{v \in X} \rho_{\vec{G}}(v) \geq i(X)$ holds for any finite set $X \subseteq V$. In order to prove that there is no $l$-deficient set, pick any 
queue $\mathcal{U}_{\theta}:=\left(U_{\lambda}\right)_{\lambda \leq \theta}$. We will show by transfinite induction that $\eta\left(\mathcal{U}_{\lambda}, l\right) \geq \delta_{\vec{G}}\left(U_{\lambda}\right)$ for every $\lambda \leq \theta$. (Recall that $\delta_{\vec{G}}(U)$ denotes the number of edges leaving $U$.) This is true for $\lambda=0$. Let $\lambda$ be the smallest ordinal for which this is not yet shown.

First, let $\lambda$ be a successor ordinal, and assume that $\sum_{v \in U_{\lambda}^{\prime}} \rho_{\vec{G}}(v)<\infty$. Then

$$
\begin{aligned}
\eta\left(\mathcal{U}_{\lambda}, l\right) & =\eta\left(\mathcal{U}_{\lambda-1}, l\right)+i\left(U_{\lambda}^{\prime}\right)+d\left(U_{\lambda}^{\prime}, \overline{U_{\lambda}}\right)-l\left(U_{\lambda}^{\prime}\right) \\
& \geq \delta_{\vec{G}}\left(U_{\lambda-1}\right)+i\left(U_{\lambda}^{\prime}\right)+d\left(U_{\lambda}^{\prime}, \overline{U_{\lambda}}\right)-\sum_{v \in U_{\lambda}^{\prime}} \rho_{\vec{G}}(v) \\
& =\delta_{\vec{G}}\left(U_{\lambda-1}\right)+d\left(U_{\lambda}^{\prime}, \overline{U_{\lambda}}\right)-\rho_{\vec{G}}\left(U_{\lambda}^{\prime}\right)=\delta_{\vec{G}}\left(U_{\lambda}\right) .
\end{aligned}
$$

If, on the other hand, $\sum_{v \in U_{\lambda}^{\prime}} \rho_{\vec{G}}(v)=\infty$ then either there are infinitely many edges directed from $U_{\lambda-1}$ to $U_{\lambda}^{\prime}$, in which case $\eta\left(\mathcal{U}_{\lambda-1}, l\right) \geq \delta_{\vec{G}}\left(U_{\lambda-1}\right)=\infty$, or $i\left(U_{\lambda}^{\prime}\right)=\infty$, or there are infinitely many edges directed from $\overline{U_{\lambda}}$ towards $U_{\lambda}^{\prime}$, which implies $d\left(U_{\lambda}^{\prime}, \overline{U_{\lambda}}\right)=\infty$. In all of these cases we obtain

$$
\eta\left(\mathcal{U}_{\lambda}, l\right)=\eta\left(\mathcal{U}_{\lambda-1}, l\right)+i\left(U_{\lambda}^{\prime}\right)+d\left(U_{\lambda}^{\prime}, \overline{U_{\lambda}}\right)-l\left(U_{\lambda}^{\prime}\right) \geq \infty-\infty=\infty .
$$

Next, let $\lambda$ be a limit ordinal. Denoting by $A(X, Y)$ the edges directed from $X \subseteq V$ to $Y \subseteq V$ we obtain

$$
\begin{aligned}
\eta\left(\mathcal{U}_{\lambda}, l\right) & =\lim \inf _{\mu<\lambda} \eta\left(\mathcal{U}_{\mu}, l\right) \geq \lim \inf _{\mu<\lambda}\left(\delta_{\vec{G}}\left(U_{\mu}\right)\right) \\
& \geq \lim \inf _{\mu<\lambda}\left|A\left(U_{\mu}, \overline{U_{\lambda}}\right)\right|=\delta_{\vec{G}}\left(U_{\lambda}\right) .
\end{aligned}
$$

Finally, with $\lambda=\theta$ we get $\eta\left(\mathcal{U}_{\theta}, l\right) \geq \delta_{\vec{G}}\left(U_{\theta}\right) \geq 0$, as desired.

\section{Open questions}

Let us formulate two directions for future research. First, Theorem 2 treats only countable graphs, and indeed our proof does not seem to be adaptable to higher cardinalities. On the other hand, we do not have any example showing that our condition fails in uncountable graphs.

Problem 8. Can Theorem 2 be extended to uncountable graphs?

Second, in finite graphs, Theorem 1 allows to impose lower bounds on the in-degree and the out-degree at the same time. Indeed, $d(v)-u(v)$ gives a lower bound on the outdegree of a vertex $v$. In contrast, for a vertex $v$ of infinite degree we can only demand all or nothing. Setting $u(v)$ to a finite value in Theorem 2 is the same as requiring infinitely many outgoing edges at $v$, whereas putting $u(v)=\infty$ will not impose any restrictions on the out-degree at all. To regain a finer control, we propose the following conjecture: 
Conjecture 9. Let $G$ be a countable graph, and let $l, r: V(G) \rightarrow \mathbb{N} \cup\{\infty\}$ be two non-negative functions with $l(v)+r(v) \leq d(v)$ for all vertices $v$. Then

(i) there exists an orientation $\vec{G}$ of $G$ such that $\rho_{\vec{G}}(v) \geq l(v)$ and $\delta_{\vec{G}}(v) \geq r(v)$ for each vertex $v$

if and only if

(ii) there are no l-deficient sets and no r-deficient sets.

\section{References}

[1] A.R. Berg and T. Jordán, Algorithms for graph rigidity and scene analysis, Preprint 2003.

[2] R. Diestel, Graph theory (3rd edition), Springer-Verlag, 2005.

[3] L. Egyed, Ueber die wohlgerichteten unendlichen Graphen, Math. phys. Lapok 48 (1941), 505-509, in Hungarian with German summary.

[4] L.R. Ford and D.R. Fulkerson, Flows in networks, Princeton Univ. Press, 1962.

[5] A. Frank, On disjoint trees and arborescences, Algebraic Methods in Graph Theory (Szeged 1978), vol. 25, Soc. J. Bolyai, 1978, pp. 402-412.

[6] _ Applications of submodular functions, Surveys in Combinatorics (K. Walker, ed.), Cambridge Univ. Press, 1993.

[7] A. Frank and A. Gyárfás, How to orient the edges of a graph, Coll. Math. Soc. J. Bolyai 18 (1976), 353-364.

[8] C.St.J.A. Nash-Williams, On orientations, connectivity and odd pairings in finite graphs, Canad. J. Math. 12 (1960), 555-567.

[9] _ Another criterion for marriage in denumerable societies, Ann. Disc. Math. 3 (1978), 165-179.

[10] H.E. Robbins, A theorem on graphs with an application to a problem on traffic control, American Math. Monthly 46 (1939), 281-283.

[11] C. Thomassen, Configurations in graphs of large minimum degree, connectivity or chromatic number, Combinatorial mathematics (Proc. 3rd Int. Conf. 1985), vol. 555, Ann. N.Y. Acad. Sci., 1989, pp. 402-412.

[12] J. Wojciechowski, A necessary condition for the existence of disjoint bases of a family of infinite matroids, Congr. Numerantium 105 (1994), 97-115. 